\title{
Postural stability during standing and its association with physical and cognitive functions in non-dialysis chronic kidney disease patients
}

\author{
Thomas J. Wilkinson ${ }^{1,2}$ (1) . Daniel G. D. Nixon ${ }^{1,2} \cdot$ Alice C. Smith $^{1,2}$
}

Received: 28 February 2019 / Accepted: 4 June 2019 / Published online: 18 June 2019

(c) The Author(s) 2019

\begin{abstract}
Purpose Chronic kidney disease (CKD) is characterised by poor physical function. A possible factor may be aberrant changes to balance and postural stability (i.e. ability to maintain centre of pressure (COP)). Previous research has exclusively focused on patients undergoing renal replacement therapy (RRT). The current study investigated postural stability in a group of CKD patients not requiring RRT.

Methods 30 CKD patients (aged $57.0 \pm 17.8$ years, $47 \%$ female, mean eGFR $42.9 \pm 27.2 \mathrm{ml} / \mathrm{kg} / 1.73 \mathrm{~m}^{2}$ ) underwent a series of physical function assessments including the sit-to-stand-5 and -60, incremental shuttle walk test, gait speed, and short physical performance battery. Postural stability (defined as total COP ellipse $\left(\mathrm{mm}^{2}\right)$ displacement) was measured using the Fysiometer board. Control reference data were provided by the manufacture. Cognitive function was assessed using the 'Montreal Cognitive Assessment-Basic' (MOCA-B)'.

Results CKD patients had poorer postural stability during quiet standing than reference values across all age categories $\left(\leq 39\right.$ years, $24.9 \pm 11.3$ vs. $10.4 \pm 1.8 \mathrm{~mm}^{2} ; 40-59$ years, $34.3 \pm 19.0$ vs. $17.7 \pm 6.2 \mathrm{~mm}^{2} ; \geq 60$ years, $39.7 \pm 21.2$ vs. $16.8 \pm 2.9 \mathrm{~mm}^{2}$, all comparisons $\left.P<0.001\right)$. Reductions in postural stability were associated with both physical and cognitive functioning. In females only, postural stability worsened with declining renal function $(r=-0.790, P<0.01)$.

Conclusions To our knowledge, this is the first and largest experimental report concerning measurement of postural stability of CKD patients not requiring RRT. Our findings suggest that postural stability is associated with worse physical and cognitive functioning in this patient group.
\end{abstract}

Keywords Postural instability $\cdot$ Balance $\cdot$ Chronic kidney disease $\cdot$ Physical function $\cdot$ Cognitive function

\section{Introduction}

Chronic kidney disease (CKD) is characterised by poor physical function that concomitantly declines with disease progression [1]. In patients not requiring renal replacement therapy (RRT), along with reducing quality of life and the ability to conduct activities of daily living, poor physical

Electronic supplementary material The online version of this article (https://doi.org/10.1007/s11255-019-02192-4) contains supplementary material, which is available to authorized users.

Thomas J. Wilkinson

t.j.wilkinson@leicester.ac.uk

1 Leicester Kidney Lifestyle Team, Department of Health Sciences, University of Leicester, Leicester LE1 7RH, UK

2 NIHR Leicester Biomedical Research Centre, Leicester LE5 4PW, UK functioning is a significant prognosticator of mortality, adverse clinical outcomes [2] and falls [3].

Centre of pressure (COP) is the point of application of the resultant force between the feet and ground [4]. Postural stability is defined as the body's ability to maintain COP relative to the base of support, either in a fixed position or during movement such as standing and walking [5]. Previous research into postural stability in renal populations has exclusively focused on RRT patients. Shin et al. [6] reported that under static balance conditions, haemodialysis (HD) patients exhibited lower postural stability compared to healthy individuals, whilst Blake et al. [7] reported postural stability was 39\% poorer in HD patients than controls. Similar findings have been observed in renal transplant recipients (RTRs) [8]. In HD patients, lower postural stability is associated with poor self-reported physical function [7], and is considered an important cause of falls in the elderly [8, 
9]. No research has investigated postural stability in nondialysis-dependent CKD patients.

Postural stability is the product of a complex interplay of the sensory information processing (i.e. visual, vestibular, and proprioceptive) and motor output. Impairment to these processes can lead to decreased control of posture $[7,8$, 10]. In renal patients $[6,8]$ and the elderly [11], impairment to postural stability may be a result of muscle atrophy and weakness [6] which reduces proprioception efficiency [8]. Along with physical functioning, there is also evidence that mobility and balance are associated with cognitive processing $[6,10]$. In HD patients, postural stability was reduced by the addition of the performance of a simultaneous cognitive task [6]. It is thought that postural stability could be reduced by impaired visuospatial function or visual memory [12].

Evaluating postural stability in patients is clinically important as it is a known risk factor for falls $[8,9]$, and associated with reductions in poor cognitive processing [6] and self-reported physical function [7]. Early identification of patients with poor postural stability may allow appropriate intervention. Given that postural stability is reduced in other renal populations, it appears important for further investigation into the prevalence of poor postural stability and factors associated with it in patients with non-dialysisdependent CKD. The objectives of the current study were to: (1) investigate postural stability in CKD compared to control reference values; (2) explore the association of postural stability with physical function; (3) explore the relationship between postural stability and cognitive functioning; and (4) investigate potential variables that contribute to postural stability. We hypothesised that CKD patients would have significantly poorer postural stability that a control reference cohort, and that poor postural stability would be associated with reductions in physical and cognitive functioning.

\section{Materials and methods}

This is a sub-analysis of postural stability data collected from CKD patients not requiring RRT as part of a larger cross-sectional study (registered prospectively as ISRCTN11615440) looking at cardiovascular risk and physical condition in kidney patients. Patients attended Leicester General Hospital, UK, for a single visit where outcome measures were taken.

\section{Participants}

Patients attending nephrology outpatient clinics based at the Leicester General Hospital, UK, were recruited to take part. Eligible patients were aged $\geq 18$ years with diagnosed CKD not requiring RRT. Patients were excluded if they were pregnant, or had difficulties that limited provision of informed consent. Ethical approval was sought from the NRES Committee East Midlands-Derby (14/EM/1049) and the study was conducted in accordance with the Helsinki Declaration. Informed consent was obtained from all individual participants included in the study.

\section{Outcomes}

\section{Postural stability}

Postural stability was assessed using a FysioMeter device. This is a modified Nintendo Wii Balance board (Nintendo, Kyoto, Japan) that contains four transducers used to assess force distribution and resultant COP displacement. 16-bit digital data samples at approximately $100 \mathrm{~Hz}$ were transferred via Bluetooth technology to custom-made software on a portable computer (FysioMeter ApS, Brønderslev, Denmark). The data were filtered using a fourth-order Butterworth filter with a cut-off frequency of $20 \mathrm{~Hz}$. The device has been validated against a laboratory-grade force platform (AMTI Model OR6-5, Watertown, USA) in tasks of varying difficulty [13] and used in a variety of clinical populations $[9,10]$.

Patients stood bipedal on the board with their feet shoulder-width apart and their eyes open. Patients were instructed to stand quietly for $30 \mathrm{~s}$ whilst keeping their head facing forward. Total COP ellipse area $\left(\mathrm{mm}^{2}\right)$, representing the sum of postural sway in the anteroposterior and mediolateral directions, was tracked. The average of three attempts was taken with an inability to stand for the full duration resulting in no score. Greater body sway (i.e. poorer stability of COP) resulted in greater COP ellipse area. Figure 1 shows the device and COP ellipse area output.

Using the same protocol, comparative control reference data were taken from the normative values of 354 adults (aged 20-99 years) provided online by the manufacturers (FysioMeter ApS). Data for the reference cohort were divided into the pre-defined age categories for each gender. The average age of this cohort was 55.1 years ( $41 \%$ males). Full characteristics of this group are reported elsewhere [14].

\section{Basic demographic and clinical information}

Demographic (age, sex, ethnicity) and clinical (co-morbidity, renal function, latest haematology and blood chemistry counts, medication, disease aetiology) data were taken from medical records.

\section{Physical function}

To assess objective physical function, several tests were used: 
Fig. 1 Fysiometer device and example trace for COP output. Red line shows changes in COP trace. Total COP ellipse area, representing the sum of postural sway in the anteroposterior and mediolateral directions, is calculated after $30 \mathrm{~s}$ of standing

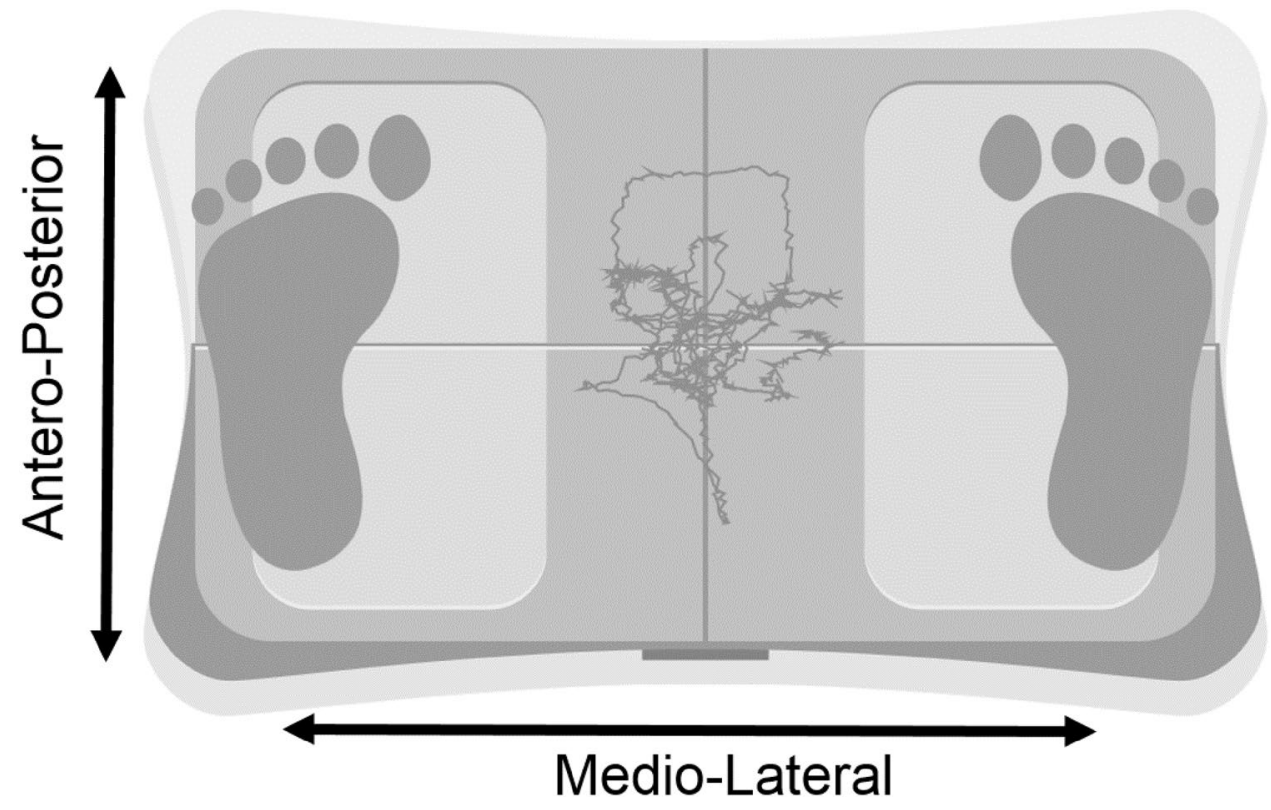

- The 'sit-to-stand' (STS)-5 and -60 tests were used as measures of lower body strength and muscle endurance [15]. Sitting on a seat ( $43.2 \mathrm{~cm}$ from the ground) with their hands across their chest, patients were asked to: (1) perform five complete STS cycles as fast as possible (STS-5); and (2) perform as many STS cycles in $60 \mathrm{~s}$ (STS-60).

- Usual gait speed was measured over a marked 4-m course, with the faster of two trials used for analysis. Gait speed is a well-established predictor of mortality in CKD [2]. A gait speed $\leq 0.8 \mathrm{~m} / \mathrm{s}$ was deemed as poor due to its association with increased mortality in this population [2].

- The 'Incremental Shuttle Walk Test' (ISWT) was used to measure cardiorespiratory walking and exercise capacity. The ISWT is a symptom-limited maximally progressive test that involves the patient walking a total of $10 \mathrm{~m}$ back and forth, and around two cones. Walking pace, through a series of bleeps, is externally dictated [16]. We have recently validated this test in CKD [17].

- The 'Short Physical Performance Battery' (SPPB) is a well-established measure of lower extremity function, previously used in CKD research [18]. A total score (/12) is derived from: (1) usual $4 \mathrm{~m}$ gait speed; (2) tests of standing balance (side-by-side, semi-tandem, tandem positions); and (3) STS-5. A five-level categorical score was assigned to each test; 0 representing inability to complete the test and 4 representing the highest level of performance [19]. A total SPPB score $<10$ was deemed as poor due to its association with increased mortality in this population [2].

\section{Cognitive function}

The 'Montreal Cognitive Assessment-Basic' (MOCA-B) was used to test cognitive impairment. A validated 30-point test, the MOCA-B evaluates six cognitive domains: visual perception (superimposed objects), executive functioning (alternating trail making; word similarity; problem-solving), language (fruit fluency; animal naming), attention (modified digit Stroop), memory (five-word recall), and orientation (time and place) [20].

\section{Body composition}

To assess the effect of body composition, we measured skeletal muscle mass and body fat using multi-frequency bioelectrical impedance analysis (InBody 370, CA, USA). Both absolute (kg) and relative (\% of total body mass) body composition values were calculated. Our group has validated this device in a cohort of non-dialysis-dependent CKD patients and RTRs [21].

\section{Statistical analysis}

Demographic data are shown as mean $( \pm \mathrm{SD})$ or $n(\%)$. As per manufacture settings, control reference data were categorised into the age ranges $\leq 39(n=103), 40-59$ $(n=87), \geq 60(n=164)$. Independent samples $T$ tests were used to investigate the differences between CKD and reference data. The difference between CKD and reference data is expressed as a \% of the CKD data. Distribution of COP was normally distributed (assessed using 
Shapiro-Wilk test) across three age categories ( $\leq 39$ years, 40-59 years, $>60$ years) in the CKD group.

Bivariate and partial correlations were used to explore the association between postural stability, and physical and cognitive function. For all associations, an unadjusted bivariate analysis (Model 1) and partial correlation analysis adjusted for age, sex, ethnicity, BMI, and eGFR (Model 2) were performed. Potential predictive factors of postural instability were analysed using forced entry linear regression modelling. Regression models were run using both absolute and relative body composition variables. The number of missing data is shown as supplementary material 1. Statistical significance was recognised as $P<0.05$. Data analysis was performed on IBM SPSS Statistics 24 (NY, USA) and Prism 7 (CA, USA).

\section{Results}

Basic characteristics of patients consented to the study are shown in Table 1. Overall, the mean age was $57.0 \pm 17.8$ years old with $47 \%$ of the cohort female. The mean eGFR was $42.9 \pm 27.2$ [min: 8 , max: 90 ] ml/ $\mathrm{kg} / 1.73 \mathrm{~m}^{2}$ with the majority of patients $(74 \%)$ in CKD stages $3 \mathrm{a}-5$. No patients required RRT. The mean age and gender distribution was similar to the control reference cohort. The functional status of our CKD sample was poor with eight (27\%) patients recording a SPPB score $<10$ and two (7\%) patients having a gait speed $\leq 0.8 \mathrm{~m} / \mathrm{s}$.

\section{Postural stability in CKD versus control reference data}

Postural stability was significantly lower in CKD than the reference cohort across all age ranges (Fig. 2). The mean COP ellipse area for CKD patients aged $\leq 39$ years was $24.9 \pm 11.3 \mathrm{~mm}^{2}$ compared to $10.4 \pm 1.8 \mathrm{~mm}^{2}$ for the reference cohort $(-58 \%, P<0.001)$. In CKD patients aged 40-59 years, the mean COP ellipse area was $34.3 \pm 19.0 \mathrm{~mm}^{2}$ compared to $17.7 \pm 6.2 \mathrm{~mm}^{2}$ for the reference cohort $(-48 \%, P<0.001)$, and the mean COP ellipse area for CKD patients $\geq 60$ years was $39.7 \pm 21.2 \mathrm{~mm}^{2}$ compared to $16.8 \pm 2.9 \mathrm{~mm}^{2}$ for the reference cohort $(-58 \%, P<0.001)$. There was no statistically significant difference between CKD patients aged $\leq 39$ and $\geq 60$ years $(P=0.12)$, or those aged $40-59$ and $\geq 60(P=0.57)$. CKD patients aged $\leq 39$ had $33 \%$ poorer postural stability than the reference cohort aged $\geq 60$ years $(P<0.001)$.
Table 1 Patient characteristics

\begin{tabular}{|c|c|}
\hline & $n=30$ \\
\hline Age (years) & $57.0 \pm 17.8$ \\
\hline Sex, $n$ female $(\%)$ & $14(47)$ \\
\hline Height (cm) & $170.4 \pm 8.2$ \\
\hline Body mass (kg) & $86.0 \pm 16.5$ \\
\hline Body mass index $\left(\mathrm{kg} / \mathrm{m}^{2}\right)$ & $29.5 \pm 4.8$ \\
\hline Body fat (\%) & $37.5 \pm 5.3$ \\
\hline Waist circumference $(\mathrm{cm})$ & $101.3 \pm 13.8$ \\
\hline Hip circumference $(\mathrm{cm})$ & $104.6 \pm 31.5$ \\
\hline Hip to waist ratio & $1.3 \pm 1.7$ \\
\hline \multicolumn{2}{|l|}{ Ethnicity } \\
\hline White British, $n(\%)$ & $24(80)$ \\
\hline Asian, $n(\%)$ & $5(17)$ \\
\hline Caribbean, $n(\%)$ & $1(3)$ \\
\hline \multicolumn{2}{|l|}{ Clinical parameters } \\
\hline Systolic blood pressure (mmHg) & $133 \pm 15$ \\
\hline Diastolic blood pressure $(\mathrm{mmHg})$ & $80 \pm 13$ \\
\hline eGFR $\left(\mathrm{ml} / \mathrm{kg} / 1.72 \mathrm{~m}^{2}\right)$ & $42.9 \pm 27.2$ \\
\hline Haemoglobin $(\mathrm{g} / \mathrm{dl})$ & $12.9 \pm 1.6$ \\
\hline \multicolumn{2}{|l|}{ Disease aetiology } \\
\hline Diabetic nephropathy, $n(\%)$ & $5(17)$ \\
\hline IgA nephropathy, $n(\%)$ & $5(17)$ \\
\hline Polycystic kidney disease, $n(\%)$ & $3(10)$ \\
\hline Primal focal segmental glomerulosclerosis, $n(\%)$ & $2(7)$ \\
\hline Other, $n(\%)$ & $4(12)$ \\
\hline Unknown/not stated, $n(\%)$ & $11(37)$ \\
\hline \multicolumn{2}{|l|}{ Co-morbidities } \\
\hline Diabetes, $n(\%)$ & $9(30)$ \\
\hline (Diagnosis of) hypertension, $n(\%)$ & $21(70)$ \\
\hline
\end{tabular}

Unless stated otherwise, data presented as mean $( \pm \mathrm{SD})$ $e G F R$ estimated glomerular filtration rate

\section{Postural stability and physical functioning}

Table 2 shows the association between postural stability and physical function. In an unadjusted model and adjusted model 2 (controlling for age, sex, ethnicity, BMI, and eGFR), reductions in postural stability were significantly associated with poorer physical function scores (all tests). Reductions in postural stability were strongly associated with an increased time to complete the STS- 5 test $(r=0.846$, $0.869, P<0.01$ across all models).

\section{Postural stability and cognitive function}

The average MOCA-B score was $27.6 \pm 2.8$ with $29 \%$ of patients scoring the maximum of 30 points. Poorer postural stability was strongly correlated with a lower MOCA-B 
Fig. 2 Differences in postural stability between CKD patients and control reference data. Postural instability defined as using total COP ellipse area $\left(\mathrm{mm}^{2}\right)$, representing the sum of postural sway in the anteroposterior and mediolateral directions, over 30 s. $C K D$ chronic kidney disease

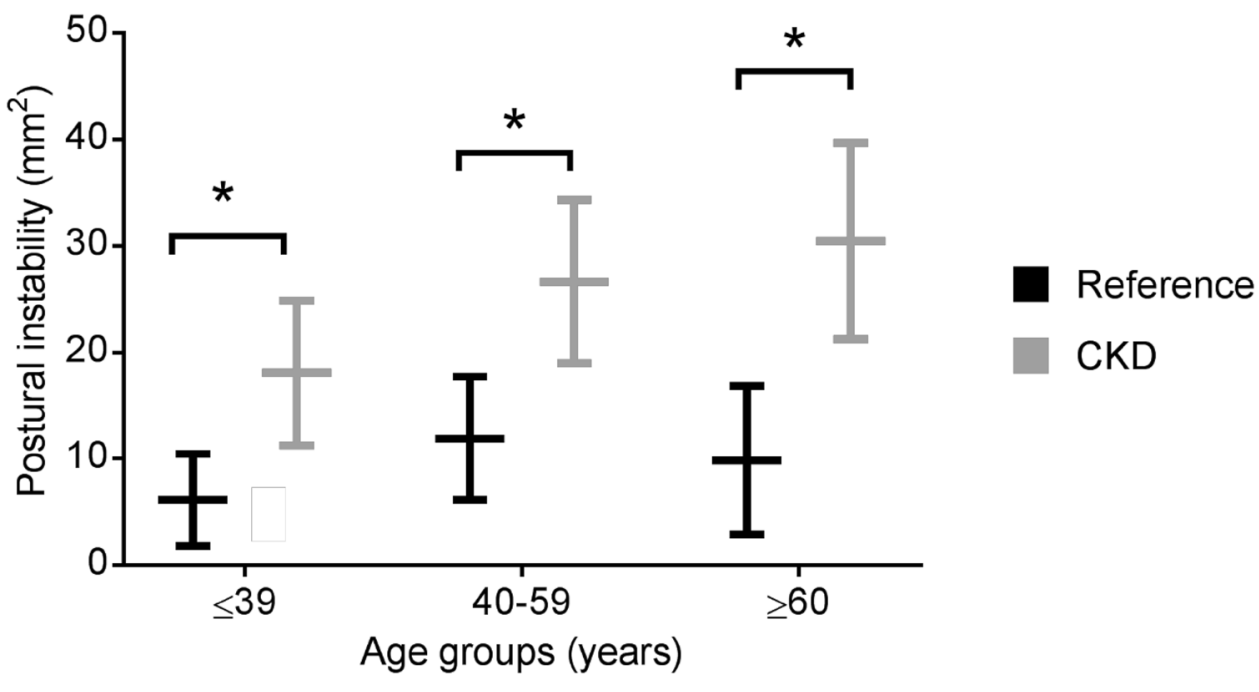

Table 2 The association of postural stability and physical and cognitive function

\begin{tabular}{|c|c|c|c|c|}
\hline & \multicolumn{2}{|c|}{$\begin{array}{l}\text { Model } 1 \text { (unad- } \\
\text { justed) }\end{array}$} & \multicolumn{2}{|c|}{$\begin{array}{l}\text { Model } 2 \text { (unad- } \\
\text { justed) }\end{array}$} \\
\hline & $r$ & $P$ & $r$ & $P$ \\
\hline STS-5 (s) & 0.846 & $<0.01 *$ & 0.869 & $<0.01 *$ \\
\hline STS-60 (repetitions) & -0.481 & $0.01 *$ & -0.500 & $0.04 *$ \\
\hline Gait speed $(\mathrm{m} / \mathrm{s})$ & 0.624 & $<0.01 *$ & -0.500 & $0.04 *$ \\
\hline ISWT (m) & -0.622 & $<0.01 *$ & -0.488 & $0.05^{*}$ \\
\hline SPPB (total score) & -0.707 & $<0.01 *$ & -0.674 & $0.03 *$ \\
\hline MOCA-B (total score) & -0.770 & $<0.01 *$ & -0.723 & $0.01 *$ \\
\hline
\end{tabular}

Significant values are in bold

Model 2 =adjusted for age, sex, ethnicity, BMI, and eGFR

eGFR estimated glomerular filtration rate, BMIbody mass index, $S T S$ sit-to-stand, ISWT incremental shuttle walk test, $S P P B$ short physical performance battery, MOCA-B Montreal Cognitive AssessmentBasic. Significance set at $<0.05$

score (Model 1: $r=-0.770, P<0.01$; Model 2: $r=-0.723$, $P=0.01$ ) (Table 2).

\section{Factors influencing postural instability}

Postural stability worsened with declining renal function (eGFR), although this was statistically significant in female patients only (male, $r=0.259, P=0.39$; female, $r=0.790$, $P<0.01$ ) (Fig. 3). The only significant predictor variable of postural stability was greater absolute skeletal muscle mass

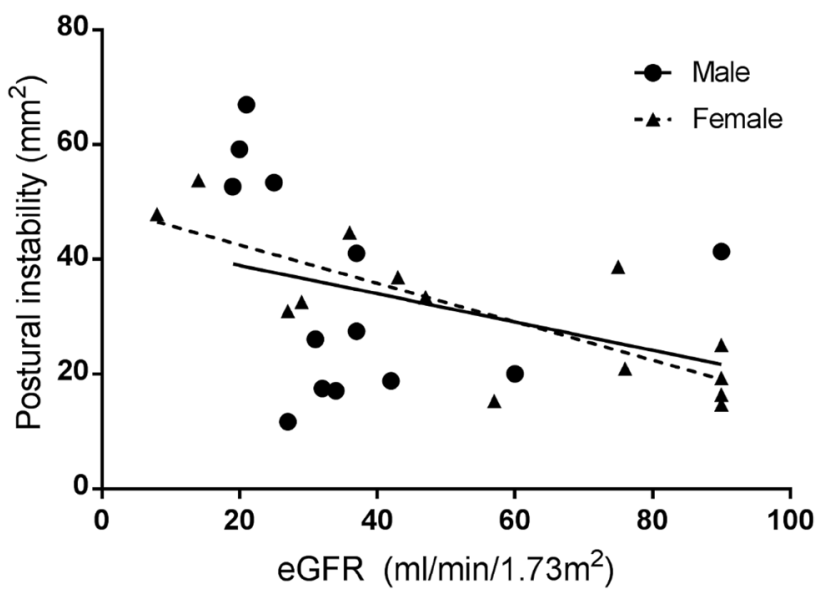

Fig. 3 Relationship between renal function and postural instability in male and female CKD patients. Postural instability defined as using total COP ellipse area $\left(\mathrm{mm}^{2}\right)$, representing the sum of postural sway in the anteroposterior and mediolateral directions, over $30 \mathrm{~s}$. $C K D$ chronic kidney disease, $e G F R$ estimated glomerular filtration rate

$(\beta=1.301, P=0.03)$. Relative muscle was not significant predictors of postural stability (Table 3 ).

\section{Discussion}

The primary findings of the current study were that: (1) CKD patients have poorer postural stability than a control reference cohort; (2) reduced postural stability is associated with poor physical and cognitive functioning; and (3) postural stability worsens with declining renal function. To our knowledge, this is the first experimental report concerning measurement of postural stability in this population. 
Table 3 Potential predictive factors of postural stability in male and female CKD patients

\begin{tabular}{|c|c|c|c|c|c|}
\hline & $B$ & $95 \% \mathrm{CI}$ & $\beta$ & $t$ & $P$ \\
\hline \multicolumn{6}{|l|}{ Absolute body composition } \\
\hline Age (years) & 0.038 & -1.022 to 1.098 & 0.017 & 0.075 & 0.94 \\
\hline Sex & 17.552 & -24.878 to 59.982 & 0.219 & 0.858 & 0.40 \\
\hline eGFR (ml/kg/1.72 m²) & -0.466 & -1.221 to 0.289 & -0.306 & -1.279 & 0.21 \\
\hline Skeletal muscle mass $(\mathrm{kg})$ & 3.542 & 0.314 to 6.771 & 0.570 & 2.275 & $0.03 *$ \\
\hline Body fat (kg) & -0.527 & -2.061 to 1.006 & -0.163 & -0.713 & 0.48 \\
\hline \multicolumn{6}{|l|}{ Relative body composition } \\
\hline Age (years) & 0.241 & -1.035 to 1.517 & 0.105 & 0.391 & 0.70 \\
\hline Sex & -10.059 & -49.451 to 29.333 & -0.126 & -0.530 & 0.60 \\
\hline eGFR (ml/kg/1.72 $\left.\mathrm{m}^{2}\right)$ & -0.425 & -1.262 to 0.411 & -0.279 & -1.055 & 0.30 \\
\hline Skeletal muscle mass (\%) & -1.945 & -20.678 to 16.788 & -0.185 & -0.215 & 0.83 \\
\hline Body fat (\%) & -0.858 & -12.363 to 10.647 & -0.131 & -0.155 & 0.88 \\
\hline
\end{tabular}

Significant values are in bold

$e G F R$ estimated glomerular filtration rate. Significance set at $<0.05$
Using total COP ellipse area during a 30 -s quiet standing task as measure of postural stability, we found that patients exhibited significantly greater displacement than a control reference cohort. On average, the postural stability was between $48 \%$ and $58 \%$ poorer than the reference values. In 19 patients undergoing HD treatment, Shin et al. [6] showed that during quiet standing, total sway area was $88 \%$ larger than healthy controls. In that study, anteroposterior displacement was 38\% larger and mediolateral displacement 54\% greater. Blake et al. [7] showed that anteroposterior displacement was 39\% greater in 12 patients undergoing HD than healthy controls. Here, postural sway was measured over a 10 -s period with patients demonstrating impairments under eyes open and closed conditions. Interestingly, balance deteriorated more than controls when visual input was eliminated and greater reliance was placed on the somatosensory and vestibular systems; the authors proposed that elevated postural sway was a result of proprioceptive dysfunction. In 19 RTRs, Zanotto et al. [8] found that during $30 \mathrm{~s}$ of quiet standing, RTRs had greater anteroposterior displacement $(22 \%)$ than healthy controls. The authors postulated that increased postural sway may indicate an impaired capacity to rely on proprioceptive and vestibular information. Unfortunately, no data relating to proprioceptive function were recorded in our current study.

Reduced postural stability was associated with poor physical function in our group. In particular, postural stability was strongly associated with the time taken to complete the STS-5 and SPPB test. Given the importance of balance in standing from a seated position, this result is perhaps expectable. This suggests that efforts to improve postural stability may be a key factor in improving physical function in this group. Research into the relationship between postural stability and physical function is limited. In HD patients, greater COP displacement was associated with poor selfreported physical function as measured using the SF-36 questionnaire [7]. In patients with osteoarthritis, decreased postural control (assessed using a one-leg stand test) was associated with a longer time in the 'Get Up and Go' test [22], and in healthy middle-aged participants, greater anteroposterior body sway was associated with poorer gait speed [23].

We identified a strong significant relationship between postural stability and cognitive function as measured by the MOCA-B. The MOCA-B is a validated measure of cognitive impairment and includes assessment of visual perception, executive functioning, and memory. It has been suggested that postural stability could be reduced by impaired visuospatial function or visual memory [12]. Murakami et al. [24] showed in Parkinson's disease patients, poor postural stability (measured by the Unified Parkinson's Disease Rating Scale) was associated with reduced MOCA scores, particularly the visuospatial and orientation subdomains. It is thought that the postural control system integrates sensory information and produces motor commands in response to environmental changes [24]. This level of central motor processing also includes visuospatial and executive functions, and motor and cognitive impairments may share a common pathophysiology (e.g. neural pathways) [6, 24]. With the MOCA-B acting as a 'proxy' indicator of visuospatial and executive function, which is important in posture [12], this may explain the association in our data.

Previous research has suggested that in renal patients [6, $8]$ and the elderly [11], reductions in postural stability may be the result of aberrant body composition. Unlike previous work $[6,8,25]$, we found no association between poor postural stability and increased fat mass. However, we did observe reduced postural stability in those with greater absolute muscle mass. This contrasts research by Zanotto et al. 
[8] who postulated that muscle atrophy reduced proprioception efficiency. Adjusting muscle mass relative to total body mass removed any association with postural stability suggesting that it may be the actual load of the body, rather that the composition of muscle and fat, that is important. Indeed, an exploratory analysis revealed a significant correlation between body mass and postural instability $(r=0.47$, $P=0.01$ ). Body mass has been shown previously to positively correlate with postural sway and obesity is associated with increased postural instability [25, 26]. In our sample, it appears that the majority of this 'mass' comes from the skeletal muscle. Alonso et al. [26] suggested that greater muscle mass and a smaller support base increase sway area, and that rather than a worsening of balance may be strategy used to stabilize COP. However, although not observed in our sample, the influence of extra fat mass on increased biomechanical loadings (e.g. joint torques and muscle forces, body segment inertial parameters, sensorimotor and central nervous systems dysfunction [25]) should not be discounted. Our data suggest that reducing body mass may confer favourable effects on COP and, therefore, physical function.

Given the importance of postural stability in CKD, efforts should be made to measure it appropriately. Postural stability is preferably investigated using posturography $[9,13]$. However, such measures are often costly, problematic to locate, and require experienced personnel; therefore, this form of assessment is not often feasible. Consequently, subjective assessment, such as the Berg Balance Scale, is commonly used in CKD [27, 28]. While more feasible, they are limited by ceiling effects and an inability to distinguish small changes [29], as demonstrated in exercise trials in CKD [28]. In our current study, we used a modified Nintendo Wii Balance board (FysioMeter device) to assess COP displacement. A validated assessment of posture control [13], the FysioMeter costs a small fraction of a laboratory-grade force platform, is mass-marketed, and is portable. Accordingly, it has the potential to become a key component in physical functional assessment $[10,13]$. If reduced postural stability is detected in patients, efforts should be made to improve it. Whilst the contributing factors are complex and likely distinct to each individual, forms of specific-balance training and exercise therapy (e.g. unilateral standing) have been shown an effective modality in the rehabilitation of balance deficits in patients with various conditions (e.g. chronic ankle instability [29], older adults [30], and Parkinson's disease [31]).

Some limitations of this study should be acknowledged. First, our assessment of postural stability occurred during a single task (quiet bipedal standing, eyes open, for $30 \mathrm{~s}$ ). It would have been advantageous to assess posture during different scenarios (e.g. dual task (i.e. addition of a cognitive task) or eyes closed to remove visual information). However, it is important to recall that the large deficits seen were observed using a simple standing task; worryingly, the addition of dual tasks or removal of sensory input may exacerbate this impairment further. Second, the Fysiometer device is unable to differentiate variation in anteroposterior and mediolateral directions. However, given the strong associations with total COP ellipse area and the ease of use of the device, it is unlikely on a practical level that this detail of information is needed by healthcare professionals. Third, our study has a low sample size and as a subset-analysis of a larger study, no a priori sample size was calculated. However, our sample of $n=30$ patients is greater than previous studies investigating postural instability in renal populations (e.g. HD, $n=19$ [6], $n=12$ [7], RTRs, $n=19$ [8]). Whilst no patients in the current study had evidence of peripheral neuropathy or neuropathic conditions, this may be an important consideration in further study of postural stability in this patient group. Although our analysis is further limited in its cross-sectional data, it does provide evidence showing postural stability is associated with both physical and cognitive functioning.

\section{Conclusion}

In conclusion, CKD patients not requiring RRT have reduced postural stability during quiet standing. We have shown that this reduction in postural stability is associated with poor physical, particularly those involving standing and cognitive functioning, and postural stability worsened along with declining renal function. These findings reveal that postural stability has potential clinical implications on patient physical and cognitive functioning. Whilst it is unlikely to be routinely assessed in all patients, our data reveal that, irrespective of age, postural stability assessments should be considered in those with advancing disease, increased body mass, and those with reduced physical functioning. Further research is needed to examine the mechanisms behind postural stability, and interventions that can be used to improve it.

Funding This research was part-funded by the Stoneygate Trust and supported by the NIHR Leicester Biomedical Research Centre.

\section{Compliance with ethical standards}

Conflict of interest The authors declare that they have no conflict of interest.

Ethical standards All procedures performed in studies involving human participants were in accordance with the ethical standards of the institutional and/or national research committee and with the 1964 Helsinki declaration and its later amendments or comparable ethical standards. 
Open Access This article is distributed under the terms of the Creative Commons Attribution 4.0 International License (http://creativeco mmons.org/licenses/by/4.0/), which permits unrestricted use, distribution, and reproduction in any medium, provided you give appropriate credit to the original author(s) and the source, provide a link to the Creative Commons license, and indicate if changes were made.

\section{References}

1. Segura-Ortí E, Gordon P, Doyle J, Johansen K (2018) Correlates of physical functioning and performance across the spectrum of kidney function. Clin Nurs Res 27:579-596

2. MacKinnon HJ, Wilkinson TJ, Clarke AL et al (2018) The association of physical function and physical activity with all-cause mortality and adverse clinical outcomes in nondialysis chronic kidney disease: a systematic review. Ther Adv Chronic Dis 9:209-226

3. Wilhelm-Leen ER, Hall YN, Tamura MK, Chertow GM (2009) Frailty and chronic kidney disease: the third national health and nutrition evaluation survey. Am J Med 122:664-671

4. Simoneau GG, Ulbrecht JS, Derr JA, Becker MB, Cavanagh PR (1994) Postural instability in patients with diabetic sensory neuropathy. Diabetes Care 17:1411-1421

5. Orr R (2010) Contribution of muscle weakness to postural instability in the elderly. Eur J Phys Rehabil Med 46:183-220

6. Shin S, Chung HR, Fitschen PJ et al (2014) Postural control in hemodialysis patients. Gait Posture 39:723-727

7. Blake C, O'Meara YM (2004) Subjective and objective physical limitations in high-functioning renal dialysis patients. Nephrol Dial Transplan 19:3124-3129

8. Zanotto T, Gobbo S, Bullo V et al (2017) Balance impairment in kidney transplant recipients without concurrent peripheral neuropathy. Gait Posture 55:116-120

9. Johansson J, Nordström A, Gustafson Y, Westling G, Nordström P (2017) Increased postural sway during quiet stance as a risk factor for prospective falls in community-dwelling elderly individuals. Age Ageing 46:964-970

10. Leach JM, Mancini M, Kaye J, Hayes T, Horak F (2018) Day-today variability of postural sway and its association with cognitive function in older adults: a pilot study. Front Aging Neurosci 10:126

11. Ochi M, Tabara Y, Kido T et al (2010) Quadriceps sarcopenia and visceral obesity are risk factors for postural instability in the middle-aged to elderly population. Geriatr Gerontol Int 10:233-243

12. Lee JM, Koh S-B, Chae SW et al (2012) Postural instability and cognitive dysfunction in early Parkinson's disease. Can J Neurol Sci 39:473-482

13. Clark RA, Bryant AL, Pua Y, McCrory P, Bennell K, Hunt M (2010) Validity and reliability of the Nintendo Wii Balance Board for assessment of standing balance. Gait Posture 31:307-310

14. Blomkvist AW, Eika F, Rahbek MT et al (2017) Reference data on reaction time and aging using the Nintendo Wii Balance Board: a cross-sectional study of 354 subjects from 20 to 99 years of age. PLoS One 12:e0189598

15. Mong Y, Teo TW, Ng SS (2010) 5-repetition sit-to-stand test in subjects with chronic stroke: reliability and validity. Arch Phys Med Rehabil 91:407-413

16. Singh S, Morgan M, Hardman A, Rowe C, Bardsley P (1994) Comparison of oxygen uptake during a conventional treadmill test and the shuttle walking test in chronic airflow limitation. Eur Respir J 7:2016-2020

17. Wilkinson TJ, Xenophontos S, Gould DW et al (2018) Test-retest reliability, validation, and "minimal detectable change" scores for frequently reported tests of objective physical function in patients with non-dialysis chronic kidney disease. Physiother Theory Pract 2018:1-12

18. Reese PP, Cappola AR, Shults J et al (2013) Physical performance and frailty in chronic kidney disease. Am J Nephrol 38:307-315

19. Guralnik JM, Simonsick EM, Ferrucci L et al (1994) A short physical performance battery assessing lower extremity function: association with self-reported disability and prediction of mortality and nursing home admission. J Gerontol 49:85-94

20. Julayanont P, Nasreddine ZS (2017) Montreal cognitive assessment (MoCA): concept and clinical review. In: Larner A (ed) Cognitive screening instruments. Springer, London, pp 139-195

21. Wilkinson TJ, Richler-Potts D, Nixon DG, Neale J, Smith AC (2018) Anthropometry-based equations to estimate body composition: a suitable alternative in renal transplant recipients and patients with nondialysis dependent kidney disease? J Ren Nutr 24:1051-2276

22. Sanchez-Ramirez DC, van der Leeden M, Knol DL et al (2013) Association of postural control with muscle strength, proprioception, self-reported knee instability and activity limitations in patients with knee osteoarthritis. J Rehabil Med 45:192-197

23. Ito T, Sakai Y, Kubo A et al (2014) The relationship between physical function and postural sway during local vibratory stimulation of middle-aged people in the standing position. J Phys Ther Sci 26:1627-1630

24. Murakami H, Owan Y, Mori Y et al (2013) Correlation between motor and cognitive functions in the progressive course of Parkinson's disease. Neurol Clin Neurosci 1:172-176

25. Singh D, Park W, Levy MS, Jung ES (2009) The effects of obesity and standing time on postural sway during prolonged quiet standing. Ergonomics 52:977-986

26. Alonso AC, Luna NMS, Mochizuki L, Barbieri F, Santos S, Greve JMDA (2012) The influence of anthropometric factors on postural balance: the relationship between body composition and posturographic measurements in young adults. Clinics 67:1433-1441

27. Painter P, Marcus R (2013) Physical function and gait speed in patients with chronic kidney disease. Nephrol Nurs J 40:529

28. Hellberg M, Höglund P, Svensson P, Abdulahi H, Clyne N (2017) Decline in measured glomerular filtration rate is associated with a decrease in endurance, strength, balance and fine motor skills. Nephrology 22:513-519

29. McKeon PO, Hertel J (2008) Systematic review of postural control and lateral ankle instability, part II: is balance training clinically effective? J Athl Train 43:305-315

30. Rugelj D (2010) The effect of functional balance training in frail nursing home residents. Arch Gerontol Geriatr 50:192-197

31. Smania N, Corato E, Tinazzi M et al (2010) Effect of balance training on postural instability in patients with idiopathic Parkinson's disease. Neurorehabil Neural Repair 24:826-834

Publisher's Note Springer Nature remains neutral with regard to jurisdictional claims in published maps and institutional affiliations. 